\title{
Current management of pregnancy-associated breast cancer
}

\author{
Harry HY Yu, Polly SY Cheung *, Roland CY Leung, TN Leung, WH Kwan
}

\section{A B S T R A C T}

Pregnancy-associated breast cancer is the most common malignancy during pregnancy with an expected rise in incidence. The belief in the need for termination of pregnancy and that chemotherapy is contra-indicated during pregnancy is challenged by recent evidence. Patients can consider breastconserving surgery and sentinel lymph node biopsy with acceptably low fetal risk from radiation exposure. A range of chemotherapeutics is possible in the second trimester in terms of drug class and frequency. Hormonal therapy and monoclonal antibody therapy are contra-indicated during pregnancy and lactation. Fetal outcome after inutero exposure to chemotherapy appears similar to that in a non-pregnant population. Future pregnancy, in most situations, does not appear to be contra-indicated but a multidisciplinary and

This article was published on 26 Jun 2017 at www.hkmj.org. patient-centred approach is recommended. Fertility preservation techniques are also being developed with reported success and consequent pregnancies.

\section{Hong Kong Med J 2017;23:387-94}

DOI: $10.12809 / \mathrm{hkmj} 166049$

${ }^{1}$ HHY Yu, FCSHK, FHKAM (Surgery)

${ }^{2}$ PSY Cheung *, FCSHK, FHKAM (Surgery)

${ }^{3}$ RCY Leung, MB, ChB, MRCP (UK)

${ }^{4}$ TN Leung, FHKCOG, FHKAM (Obstetrics and Gynaecology)

${ }^{5}$ WH Kwan, FHKCR, FHKAM (Radiology)

${ }^{1}$ Department of Surgery, Ruttonjee \& Tang Shiu Kin Hospitals, Wanchai, Hong Kong

${ }^{2}$ Breast Care Centre, Hong Kong Sanatorium \& Hospital, Happy Valley, Hong Kong

Department of Medicine, Queen Mary Hospital, Pokfulam, Hong Kong

${ }^{4}$ Obstetrics \& Gynaecology Centre, Hong Kong Sanatorium \& Hospital, Happy Valley, Hong Kong

${ }^{5}$ Comprehensive Oncology Centre, Hong Kong Sanatorium \& Hospital, Happy Valley, Hong Kong

* Corresponding author: pollyc@pca.hk

\section{Introduction}

Pregnancy-associated breast cancer (PABC) is defined as the diagnosis of breast cancer during pregnancy and those occurring within 1 year postpartum. ${ }^{1}$ It is the most common malignancy associated with pregnancy and the incidence is expected to rise in high-income countries. ${ }^{2-4}$ Historically, this disease entity was thought to have a worse prognosis when compared with patients diagnosed with cancer in the non-pregnant state and termination of pregnancy was common. Here, we reviewed recent publications and summarised the most recent information.

\section{'Dual' effect of pregnancy}

Generally it is thought that pregnancy is a protective factor that lowers the risk of breast cancer development. Nonetheless, studies have found that pregnancy increases the risk of breast cancer initially following delivery and has a protective effect after a period of time. $^{3}$ The period of increased risk has been estimated to be between 10 and 15 years following a first pregnancy. The later the first pregnancy, the longer the duration of increased risk before the protective effect. ${ }^{5}$ In a Norwegian study, women who waited until aged over 35 years for their first child permanently increased their risk of breast cancer compared with nulliparous women. ${ }^{6}$

Risk of developing breast cancer decreases with multiple pregnancies, but the age at first birth remains the dominant influence on risk. ${ }^{5} B R C A 1$ and $B R C A 2$ mutation carriers are not protected by early pregnancy from malignancy, ${ }^{2}$ but they do not have an increased risk of developing PABC compared with non-carrier women. ${ }^{5}$

\section{Epidemiology}

Breast cancer is the most common malignancy to occur during pregnancy, followed by cervical cancer, melanoma, and haematological malignancy. ${ }^{7}$ In the United Kingdom, 1.3 to 2.4 cases of breast cancer in women were diagnosed per 10000 live births. ${ }^{1}$ It accounted for $3.3 \%$ to $10 \%$ of women diagnosed with pre-menopausal breast cancer. ${ }^{2,5}$

Meta-analysis has shown that risk of death is more than $40 \%$ in women with PABC compared with those with non-PABC (pooled hazard ratio $=1.44 ; 95 \%$ confidence interval, 1.27-1.63), but other epidemiological studies have shown no direct relationship between pregnancy and mortality. ${ }^{8}$ Moreover, the prognosis of patients with breast cancer during pregnancy is similar to that of non- 


\section{與懷孕相關的乳腺癌：現行的治療方法}

余學而、張淑儀、梁澄宇、梁子昂、關永康

與懷孕相關的乳腺癌是懷孕期間最常見的惡性腫瘤, 預計發病率會逐 年上升。最新的研究挑戰着一些傳統的想法, 如乳腺癌患者需終止懷 孕以及懷孕期間不能接受化療。患者可以考慮保乳手術和前哨淋巴結 活檢, 後者所接受的輻射量對於胎兒受損的風險非常低。根據藥物類 別和用藥頻率, 有一系列的化療藥物可於妊娠第二期內使用。懷孕期 和哺乳期間不能使用激素治療和單克隆抗體治療。懷孕期間因子宮內 化療藥物暴露的胎兒與非懷乃組別的結果相若。曾經接受化療的患者 在大部分情況下不會影響將來再度懷孕的機會。雖然如此, 仍建議採 用跨學科和以病人為中心的方法。生育保存技術正在發展, 當中也有 成功再度懷孕的例子 pregnant women of the same age and clinical stage at diagnosis. ${ }^{9}$ In fact, metastasis is a rare event during pregnancy, with only case series reported in the literature. ${ }^{10}$

Epidemiological data indicate that breast cancer diagnosed during lactation exhibits the most aggressive trait and elevation in cause-specific death. ${ }^{11}$ Compared with patients in the non-pregnant state or during pregnancy, patients diagnosed with breast cancer within 1 year of delivery have a shorter time to relapse $\mathrm{e}^{12}$ and increased risk of metastasis and death. ${ }^{6}$ The lactating microenvironment is a strong driver of tumour progression. Lactating stromal adipose cells express higher levels of inflammatory cytokines that are highly angiogenic and growth promoting, causing the tumour to be more aggressive. $^{11}$

Other factors proposed to be related to the poorer outcome include the change in breast tissue and the hormonal environment. There is a shorter phase of involution, an inflammatory-like process that has been suggested to have tumour-promoting properties by affecting the microenvironment and malignant potential of microtumours. ${ }^{8}$ A generally low index of suspicion of cancer in reproductiveaged women and, in particular, pregnant women has tended to delay diagnosis by approximately 1 to 2 months according to recent studies. ${ }^{8,13}$ A 1-month delay in the treatment of a primary tumour increases the risk of axillary metastases by $0.9 \%$ to $1.8 \% .^{14}$

\section{Histopathology}

As the group of affected women diagnosed with malignancy generally represents a younger population, PABC patients tend to have high-grade tumours and display lymphovascular invasion. ${ }^{2,5,9}$ They also have a lower incidence of positive hormone receptor status. ${ }^{3}$

Placental metastasis is rare but indicates poor maternal prognosis. Pathological assessment after delivery is recommended in all cases. ${ }^{2}$

\section{Diagnosis}

Of breast masses that present to a breast clinic, $80 \%$ are benign although any mass that persists for more than 2 to 4 weeks or that is associated with skin changes raises clinical suspicion. ${ }^{2,15}$ Nipple discharge and 'milk rejection' sign, ie refusal by the infant to nurse from a diseased breast, can be signs of underlying occult carcinoma, but are not frequent. ${ }^{13,14}$

Ultrasonography (USG) is the first imaging modality following clinical examination to assess a discrete lump. ${ }^{1}$ Mammography (MMG) can also supplement USG to assess extent of disease and examine the contralateral breast. Accuracy of MMG, however, is decreased in the pregnant state because of increased water content and change in the distribution of fat. Studies have shown a falsenegative rate as high as $25 \%^{2}$ with sensitivity of $63 \%$ to $78 \% .{ }^{14}$ Radiation exposure of the fetus from MMG is minimal (0.001-0.01 mGy). The International Commission on Radiological Protection (ICRP) concluded in 2007 that there was no detrimental effects of practical significance (threshold dose, 100 mGy). ${ }^{16}$ The use of magnetic resonance imaging is controversial during pregnancy, due to the challenges in discriminating malignant from physiological hypervascularisation that occurs during pregnancy. ${ }^{13}$ There are also no safety data regarding the use of gadolinium in pregnancy, ${ }^{15}$ and fetal abnormalities have been noted in rats exposed to gadolinium. ${ }^{2}$

To complete triple assessment, core needle or excision biopsy is the gold standard for tissue diagnosis. Histological grade, receptor status, and human epidermal growth factor receptor 2 (HER2) information should be obtained, just as for nonpregnant patients. ${ }^{1,2}$ Needle cytology shows good sensitivity but with a higher risk of false-positive results due to the presence of hyperproliferative cells in mammary tissue during pregnancy and lactation. ${ }^{2}$ The risk of developing a subsequent lactating fistula after biopsy is overestimated. ${ }^{15}$ Cabergoline can reduce the risk of fistula or abscess formation by suppressing lactation. ${ }^{2}$ Emptying the breast and the use of ice packs and binding before biopsy might also reduce the risk of fistula formation. ${ }^{13}$

Unless there is a strong clinical suspicion, metastatic work-up is not mandatory. ${ }^{1}$ Chest X-ray and USG of the liver are the main modalities used to look for metastases. Fetal exposure from a chest $\mathrm{X}$-ray is about $0.1 \mathrm{mGy}^{13}$

\section{Treatment}

For those who develop breast cancer during pregnancy, any treatment intervention during 
pregnancy shows a trend towards improved overall survival compared with delaying evaluation and treatment until after delivery-78.6\% overall survival for those who received treatment during pregnancy compared with $44.7 \%$ for those patients who did not. ${ }^{17}$ Management of postpartum breast cancer is similar to that for non-pregnant patients. Consideration of fetal well-being is a critical factor when making treatment decisions for the pregnant patient.

\section{Surgery}

Surgery is considered safe in all trimesters with negligible risk to the fetus. Studies, including a large review, concluded that surgery carries a similar probability of miscarriage to the background risk of spontaneous abortion, including first trimester. ${ }^{7,18}$

Mastectomy is the definitive procedure. There is a trend for favouring breast-conserving surgery as a suitable option for PABC patients, and this should be discussed with the patient whenever possible. ${ }^{19}$

As epidemiological data show a higher percentage of axillary metastases among PABC patients, axillary dissection is generally offered. ${ }^{2}$ When the tumour is diagnosed at an early stage, however, a considerable proportion of patients will have node-negative disease and therefore might benefit from sentinel lymph node biopsy (SLNB). Such procedure remains controversial ${ }^{18}$ but some studies have reported its success and safety.

The first issue to clarify in SLNB is fetal radiation exposure relating to lymphoscintigraphy. In Italy, Gentilini et $\mathrm{al}^{20}$ reviewed 26 young nonpregnant women scheduled for lymphoscintigraphy for SLNB. A single peritumoural injection of ${ }^{99 m}$ Tc-labelled human albumin colloid particles in a volume of $0.2 \mathrm{~mL}$ was administered prior to surgery. A thermoluminescent dosimeter was placed around the abdomen (epigastrium, umbilicus, and hypogastrium) to evaluate potential uptake by the fetus in different trimesters. In 23 of 26 patients, all absorbed dose measurements over the surface of the abdomen at the supposed level of a fetus were lower than the sensitivity of the dosimeter $(<10 \mu \mathrm{Gy})$. In the remaining three patients, the absorbed doses to the epigastrium, umbilicus, and hypogastrium were 40-320, 120-150, and 30-40 $\mu \mathrm{Gy}$, respectively. Another study estimated the maximal absorbed dose of radiation from SNLB by ${ }^{99 \mathrm{~m}} \mathrm{Tc}$ sulfur colloid to be $4.3 \mathrm{mGy}^{18}$

Fetal exposure of $>100$ to $200 \mathrm{mGy}$ is associated with central nervous system problems. A radiation dose that exceeds $100 \mathrm{mGy}$ can result in reduced intelligence quotient. With a dose of $10 \mathrm{mGy}$, the risk of leukaemia and cancer is $1.4 \%$, ie a $40 \%$ increase over the normal incidence. An absorbed dose of $20 \mu \mathrm{Gy}$ is comparable with 1 to 2 days of natural background radiation, 1/5000th of the threshold dose for malformation or other adverse effects. ${ }^{21}$ Of note, reports of the ICRP have shown that the most common procedures in diagnostic nuclear medicine rarely represent an indication for termination of pregnancy, and that pregnancy should not be a reason to avoid diagnostic nuclear medicine studies. ${ }^{22}$

Gentilini et $\mathrm{al}^{21}$ then reported the use of SLNB in pregnant patients from 2001 to 2007. During the period, 12 of 45 patients diagnosed with breast cancer during pregnancy were clinically nodenegative and all underwent SLNB. Lymphatic mapping was performed by ${ }^{99 \mathrm{~m} T c-\text { radiolabelled }}$ colloid lymphoscintigraphy alone with mean activity of $10 \mathrm{MBq}$ (about $1 \mu \mathrm{Gy} / \mathrm{MBq}$ ) for the first eight patients and 3-4 MBq for the later four. Hot spots were identified in all patients (10 with 1 hot spot, and two with 2 hot spots) and the mean number of excised sentinel lymph nodes (SLNs) was two (range, 1-4). Ten patients had confirmed negative SLNs and were spared axillary dissection. One patient had micrometastasis in one of the four nodes and elected not to undergo any further axillary surgery. Another patient had a confirmed metastatic SLN intraoperatively and underwent axillary clearance at the same operation (3 of 24 positive nodes). No overt axillary lymph node reappeared for patients with negative SLN. Of the 12 pregnancies, 11 babies were born with a normal weight and no malformation after an uncomplicated pregnancy. One baby underwent surgery at 3 months of age for cardiac failure due to ventricular septal defect. This had been suspected at the 21st week of gestation prior to lymphoscintigraphy at the 26th week.

Two other studies also reported the use of SLNB with ${ }^{99 \mathrm{~m} T c}$ colloid and/or blue dye (isosulfan or methylene) for pregnant patients. ${ }^{19,21}$ All 35 patients who underwent SLNB had successful mapping and surgery was performed without complications; 33 of 35 infants were healthy at delivery. One patient found herself pregnant on the day of her scheduled operation and decided to terminate her pregnancy in the first trimester after the breast surgery in order to start chemotherapy. Another child was born with a cleft palate to a mother who was a smoker with a history of methadone use.

Axillary staging provides important prognostic information and allows better local control but does not always influence the type of adjuvant therapy. With an acceptably low false-negative rate and the same capacity to detect nodal metastasis, SLNB has a lower morbidity compared with axillary lymph node dissection (lymphoedema, $5.3 \%$ vs $11.8 \%)^{21,23}$ The decrease in surgical morbidity might result in a shorter postoperative recovery, potentially allowing an earlier start for adjuvant chemotherapy. In young premenopausal patients, such treatment can be directed towards hormone insensitive tumours and might improve outcome. ${ }^{20}$ Proponents of 
SLNB also point out the possibility of performing breast-conserving surgery and SLNB under local anaesthesia to further reduce the risk of preterm labour and spontaneous miscarriage. ${ }^{20}$

There are, however, specific issues to be solved regarding SLNB use during pregnancy. Isosulfan blue and methylene blue are Food and Drug Administration pregnancy category $\mathrm{C}$ drugs, with unknown potential risks for teratogenicity. ${ }^{23}$ Anaphylaxis has been reported with the use of blue dye. ${ }^{18,24,25}$ Adverse outcomes associated with methylene blue dye include intestinal atresia and fetal demise. ${ }^{26}$

Fetal radiation exposure and successful mapping of lymphoscintigraphy depend on the administered activity and size of radiocolloid. To minimise unnecessary radiation exposure, pregnant patients should avoid contact with other patients receiving nuclear medicine therapy, eg by scheduling pregnant patients as the first procedure of the day, and keeping them in a single-bed room. Reducing the time between lymphoscintigraphy and surgery might further reduce the dose of administered radiocolloids. ${ }^{20}$

The latest European Society for Medical Oncology (ESMO) guidelines do not discourage SLNB in pregnant breast cancer patients in centres where SLNB is routinely practised in the nonpregnant setting, but discourages the use of blue dye. ${ }^{27}$ Despite weak evidence, however, the latest American Society of Clinical Oncology guidelines are still against performing SLNB during pregnancy. ${ }^{28}$

Immediate reconstruction is not recommended and should be delayed to avoid prolonged anaesthesia and to allow optimal symmetrical breast reconstruction after delivery. ${ }^{1}$

\section{Systemic chemotherapy}

Indications for chemotherapy are the same as for non-pregnant breast cancer patients. When the indication for chemotherapy is clear, it should not be delayed due to the potential detrimental effect on maternal outcome, with the exception of breast cancer diagnosed during the first trimester. ${ }^{29}$ Adjuvant chemotherapy should be started within 3 weeks of surgery in patients with hormone-negative tumours. ${ }^{2,29}$

Most chemotherapeutic agents are of low molecular weight, highly lipid soluble, and loosely protein bound. This facilitates transplacental transfer from the mother to the fetus. ${ }^{7}$ Teratogenicity is directly related to timing and dosage delivered. The most vulnerable period for fetal malformation and spontaneous abortion is 10 days to 8 weeks after conception, ie the period of organogenesis (17\%), ${ }^{2,29}$ Throughout the first trimester, chemotherapy is still considered contra-indicated due to concerns about adverse events associated with ocular formation, genitals, the haematopoietic system, and the central nervous system before the 14th week. ${ }^{1}$

Chemotherapy is regarded as safe during the second trimester. ${ }^{1}$ However, administration during the second and third trimesters is associated with increased risk of intrauterine growth restriction and low birth rate, which may be related to both tumour burden and/or the aggressive nature of the tumour, as well as the toxicity of chemotherapy. ${ }^{29}$ Changes that occur during pregnancy, such as generation of a third space (fetal-placental amniotic fluid), increased volume distribution, and changes in the metabolism and elimination of drugs, may determine different toxicity patterns that may also indirectly affect the fetus. ${ }^{15}$ General chemotherapy risks include preterm delivery, low birth weight, transient tachypnoea of the newborn, and transient neonatal leukopenia. ${ }^{2}$ The reported fetal malformation risk following chemotherapy during the second and third trimesters is $3.8 \%$, no higher than that in the general population. ${ }^{18}$ Incidence of preterm delivery for chemotherapyexposed gestational breast cancer is $5 \%$ to $8 \% .{ }^{29}$ Most children showed normal neurological development after exposure to chemotherapy in utero, although behavioural and emotional issues need further clarification and follow-up. ${ }^{29,30}$ For PABC patients, chemotherapy-induced gonadotoxicity may cause permanent amenorrhoea with complete loss of germ cells, transient amenorrhoea, menstrual irregularity, and subfertility, but this depends on the dose, agent, and patient age. ${ }^{1}$

The German Breast Group reported a multicentre study that included 197 patients who received chemotherapy during pregnancy (a total of 447 patients in 8 years). ${ }^{4}$ Overall, $50 \%$ of breast cancer patients delivered preterm, compared with $10 \%$ to $15 \%$ of the general population. Delivery before the 37th week of gestation is associated with a higher chance of side-effects, malformations, or newborn complications. Low birth weight is affected by chemotherapy exposure after adjustment for gestational age, but not by number of chemotherapy cycles $(\mathrm{P}=0.018)$. Adverse events were more common in those who received chemotherapy in utero than in those who were not exposed (31 [15\%] of 203 vs 7 [4\%] of 170 infants; $\mathrm{P}=0.00045)$. The proportion of malformations in the study was no different to that for the general population (approximately 9\%). Two fetal deaths were reported and both were exposed to chemotherapy and delivered prematurely. Neither was thought to be related to treatment-one was related to diagnosis of trisomy 18 and the other died of necrotising enterocolitis after delivery at the 31st week with weight of $1895 \mathrm{~g}$. The study concluded that although chemotherapy exposure in utero resulted in lower birth weight and more complications, the differences were not clinically significant and most likely related to premature delivery. 
Optimum use of cytotoxic drugs in pregnant patients remains undefined, particularly regarding drug selection, dosing, and dose density. ${ }^{31}$ Assessment of treatment effectiveness in pregnant patients is complex. Calculation of chemotherapy dose is uncertain for the pregnant state. Physiological changes in pregnancy can also greatly affect drug disposition.

Anthracycline-based regimens (eg epirubicin and doxorubicin) are the most widely used chemotherapeutic agents as they have a favourable safety profile when administered during pregnancy, ${ }^{29}$ although no particular preference is given for one regimen over another. ${ }^{27}$ Assessment of maternal cardiac function by echocardiogram is recommended if an anthracycline-based regimen is to be administered..$^{14}$ Studies are yet to show an increased risk of fetal cardiotoxicity secondary to in-utero exposure. ${ }^{27}$ Common side-effects include neutropenia, oral ulcers, anaphylaxis, constipation, tachycardia, and cellulitis. Fetal side-effects include low birth weight $(7.6 \%)$ and birth defects $(3.8 \%){ }^{18}$ Risk of congenital malformations is similar to that of patients not receiving chemotherapy. Long-term follow-up of children indicates that there are no sequelae associated with growth and maturation. ${ }^{9}$

Taxanes appear to be another alternative, but have not been as extensively studied as anthracyclines and most studies used small sample sizes. ${ }^{18} \mathrm{~A}$ meta-analysis showed that the addition of taxanes to anthracycline-based regimens resulted in a statistically significant reduction in the risk of relapse (relative reduction, 17\%) and death (relative reduction, $15 \%)$ for high-risk early breast cancer patients. Disease-free survival benefit was independent of oestrogen-receptor expression, degree of nodal involvement, and type of taxane used. ${ }^{32}$ Taxanes are substrates of the P-glycoprotein that is highly expressed on the maternal compartment of the placenta. P-glycoprotein protects the fetus against xenobiotics and might therefore reduce transplacental transfer of taxanes..$^{29}$ Data for baboon and human models showed that taxanes are hardly detectable in the fetus, ${ }^{4}$ and a recent overview of 50 breast cancer patients treated with taxanes showed that completely healthy neonate was born in a majority of cases ${ }^{33}$ In a review of 40 pregnant women prescribed taxanes, there were no reports of intrauterine death or congenital malformations other than one infant with pyloric stenosis. ${ }^{34}$ Another retrospective cohort study of 12 patients with breast cancer and four with ovarian cancer who were exposed to taxane-based chemotherapy during pregnancy reported a mean gestational age at delivery of 36.9 weeks and mean birth weight of $2452 \mathrm{~g}$ (interquartile range, 2155$2619 \mathrm{~g})$. One baby was diagnosed with hypertrophic pyloric stenosis at 4 weeks and underwent surgery at 6 weeks. One of a set of twins born in this study had hyperbilirubinaemia and jaundice, and was later diagnosed with Asperger's syndrome and Tourette's syndrome, while his twin exposed to the same chemotherapy is developmentally normal and excelled at school. ${ }^{32}$

Taxanes are metabolised by cytochrome P450 that increases by $50 \%$ to $100 \%$ during the third trimester, possibly resulting in a shorter half-life and higher clearance that could result in a reduced toxicity profile during pregnancy. ${ }^{29}$ Nonetheless this lowered serum concentration is a concern for drug efficacy during pregnancy. ${ }^{14}$ There are no data to analyse different taxanes for pharmacokinetics, toxicity profile, or efficacy due to small sample size. The latest ESMO guidelines also endorsed the use of taxanes during pregnancy in cases where "they are clinically indicated or the use of anthracyclines is contraindicated".27

A dose-dense chemotherapy regimen for pregnant patients is another topic of heated debate. Chemotherapy cycles were administered every 1 to 2 weeks compared with 3-weekly cycles for conventional therapy. One study compared 10 patients who received doxorubicin and cyclophosphamide dose-dense therapy during gestation with 99 patients receiving conventional chemotherapy after the first trimester, and reported completion of chemotherapy for all patients (98\% in conventional group). They had similar delivery outcomes, risk of congenital anomalies, incidence, and time to recurrence, and maternal overall survival at 3.5 years. ${ }^{35}$ Proponents suggest that dosedense chemotherapy in pregnancy may allow faster completion of chemotherapy, sufficient time for maternal recovery for delivery, ${ }^{35}$ closer pregnancy monitoring, and better toxicity profile, and no need for high-dose steroid premedication or prophylactic use of granulocyte-colony stimulating factor. ${ }^{27}$

Chemotherapy is advised to be withheld 3 weeks before delivery or after 35 weeks of gestation to minimise the risk of sepsis and haemorrhage in the mother and newborn. ${ }^{1,2}$ It allows time for fetal drug excretion via the placenta, especially for preterm babies who have a limited ability to metabolise drugs through an immature liver and kidneys. Chemotherapy can resume after adequate recovery from delivery. ${ }^{29}$ Breastfeeding is contraindicated during the treatment period but can resume 3 to 4 weeks after the last administered dose of chemotherapy. ${ }^{2}$

\section{Radiotherapy}

Radiotherapy is contra-indicated until after delivery unless it is used for life-saving issues or to preserve organ function, eg spinal cord compression. ${ }^{1}$ If radiotherapy is indicated during the pregnant state, fetal shielding should be considered or the option of elective early delivery discussed. Substituting whole- 
breast radiotherapy with partial-breast treatment is another alternative. ${ }^{2}$ Excess cancer risk to a fetus receiving radiation is 6.57 cases per 10000 children per rad ( $0.01 \mathrm{~Gy})$ per year. The typical external beam radiation dose to the breast ranges from 45 to $60 \mathrm{~Gy}$, and may result in a fetal radiation exposure of 3.9 to $15 \mathrm{rad}$ in the first trimester and up to $200 \mathrm{rad}$ in the late third trimester. ${ }^{14}$ Other risks of radiotherapy include miscarriage, teratogenicity, microcephaly, fetal growth restriction, and induction of childhood malignancy and haematological disorders.

Adjuvant radiotherapy is not considered an urgent procedure and should be postponed until after delivery. ${ }^{27}$ Delaying treatment after 12 weeks, however, can increase the likelihood of axillary metastases by $0.028 \%$ to $0.057 \%$ per day $^{2}$ and a delay over 6 months can increase the risk of local recurrence. ${ }^{27}$

\section{Hormonal therapy}

Tamoxifen is not used until after delivery. ${ }^{1}$ It is associated with oculo-auriculo-vertebral dysplasia (Goldenhar's syndrome) and ambiguous genitalia., ${ }^{218}$ Because of unknown transmission of the drug in milk, it is also contra-indicated in breastfeeding. ${ }^{1}$ Long-term effects of the drug on female offspring are unknown. ${ }^{14}$

\section{Monoclonal antibody}

Trastuzumab is contra-indicated during pregnancy due to reported adverse fetal outcomes. ${ }^{1}$ Metaanalysis showed the main adverse event to be oligohydramnios (61.1\%), with the incidence increasing with duration of treatment. ${ }^{18}$ Alteration of amniotic fluid volume is mostly attributed to the effect of trastuzumab on the fetal kidney, where the HER2 receptor is highly expressed. ${ }^{29}$ Nonetheless, if the fetus was exposed to the drug exclusively during the first trimester, all children were completely healthy at birth: transplacental transport of immunoglobulin $\mathrm{G}$ is very low early in pregnancy and increases gradually during the second trimester to reach concentrations similar to the mother by the end of gestation..$^{29}$ Oligohydramnios is reversible if the drug is stopped, with good outcome observed in the majority of cases.

Another risk of trastuzumab for the fetus is renal failure, ${ }^{9}$ but there are no reports of serious fetal cardiac effects. ${ }^{14}$ Breastfeeding is also contraindicated due to unknown transmission of the drug in milk. The drug is not associated with impaired fertility. ${ }^{1}$

\section{Supportive agents}

Serotonin antagonists and dexamethasone are the preferred antiemetics. ${ }^{1}$ Granulocyte-colony stimulating factor is recommended to minimise potential maternal and fetal problems associated with neutropenia ${ }^{1}$ and erythropoietin has been safely administered in pregnant patients. ${ }^{18}$

In-utero exposure to bisphosphonates has been shown to increase the risk of fetal skeletal anomalies and result in hypocalcaemia that may affect uterine contraction. It is suggested that these drugs be administered after delivery whenever possible. ${ }^{29}$

A summary of current recommendations regarding treatment of PABC is shown in the Table.

\section{Antenatal care}

There is no evidence of a need for additional antenatal care, but it is standard practice to establish fetal well-being by USG before any treatment. ${ }^{2,15}$ Serial fetal growth assessment should be performed every 3 to 4 weeks, or prior to each chemotherapy cycle. Other forms of assessment of fetal well-being may be beneficial, such as umbilical artery Doppler to assess the status of the placenta, Doppler of the fetal middle cerebral artery to exclude fetal anaemia, and serial fetal echocardiograms (when potential cardiotoxic drugs like anthracyclines are being

TABLE. Summary of current recommendations for the treatment of pregnancy-associated breast cancer

\begin{tabular}{ll}
\hline Treatment & Recommendation \\
\hline $\begin{array}{l}\text { Surgery } \\
\text { Breast }\end{array}$ & Mastectomy is the definitive choice but there is a trend towards breast-conserving surgery \\
\hline Axilla & $\begin{array}{l}\text { Sentinel lymph node sampling as a choice is not discouraged, as in the non-pregnant state } \\
\text { Avoid blue dye }\end{array}$ \\
\multicolumn{1}{c}{ Reconstruction } & After delivery \\
Chemotherapy & $\begin{array}{l}\text { Anthracycline-based regimen is the most common with taxane as an alternative } \\
\text { Dose-dense therapy can be considered }\end{array}$ \\
& Avoid chemotherapy 3 weeks before delivery or after 35th week \\
\hline Hormonal therapy & Contra-indicated during pregnancy and breastfeeding \\
Monoclonal antibodies & Contra-indicated due to adverse fetal outcome \\
\hline
\end{tabular}


used). Assessment of amniotic fluid volume is also necessary because it can decrease reversibly with the use of some drugs. ${ }^{15}$

Unless there is a clear oncological or obstetric indication, delivery should be delayed until after the 37th week. Morbidity and mortality in newborns are directly related to gestational age at delivery. Infants born in late preterm (34th to 35th week) have increased morbidities including perinatal death, transient tachypnoea, respiratory distress, hypoglycaemia, pulmonary hypertension, as well as long-term cognitive and behavioural morbidities. ${ }^{7}$

Vaginal delivery is preferred because it is less likely to delay initiation of chemotherapy due to lower morbidity. ${ }^{29}$ Caesarean delivery should be reserved for the usual obstetric indications. ${ }^{2}$ Deep venous thrombosis prophylaxis should be considered, as pregnancy and malignancy are both risk factors for venous thromboembolism.

\section{Termination of pregnancy}

There is no evidence to suggest that termination of pregnancy improves prognosis. ${ }^{2,10}$ Once pregnancy has occurred, induction of abortion has no impact on maternal prognosis and is therefore strongly discouraged for such purposes. ${ }^{27}$ If maternal outcomes are not negatively impacted by the pregnancyitself, continuation of pregnancy seems not only reasonable, but recommended. ${ }^{7}$ Nevertheless, in case of advance disease stage (stage III or IV) or for high-grade or aggressive primary tumours diagnosed in the early first trimester, termination of pregnancy may be considered (teratogenic risk of chemotherapy during the first trimester). ${ }^{2,15}$

\section{Future pregnancy}

There is evidence that pregnancy after breast cancer does not lead to increased risk of recurrence and may even improve survival, although these findings could be due to the 'healthy mother effect.',14 Large matched multicentre retrospective studies including more than 1000 patients confirmed that pregnancy after oestrogen receptor (ER)-positive breast cancer was not detrimental, at least during the first 5 years following pregnancy. ${ }^{27}$ The latest ESMO guidelines also "do not discourage pregnancy following breast cancer diagnosis irrespective of the ER status". ${ }^{27}$

Nonetheless, the chance of subsequent pregnancy is nearly $70 \%$ lower when compared with the general population, probably secondary to frequent treatment with gonadotoxic chemotherapy, prolonged treatment periods with tamoxifen in patients with hormone sensitive disease, and also a general misconception that pregnancy could stimulate cancer recurrence given that it is a hormonally driven disease. ${ }^{27}$ The chance of recovery of menses is higher for patients under 40 years of age and the use of taxane-based chemotherapy. ${ }^{5}$

"Consult before conceive"-a multidisciplinary approach is recommended before planning a pregnancy. Anecdotal evidence suggests a 2-year wait after treatment and a 5-year wait for recurrent stage I and II disease. ${ }^{2}$ Patients with metastatic disease are advised against pregnancy due to their limited life expectancy and possible compromised treatment of disease. ${ }^{1}$ Interruption of full-course tamoxifen may have detrimental effects on breast cancer outcome. If, however, a woman is willing to accept the risk, interruption after 2 to 3 years of tamoxifen may be considered to allow pregnancy. Tamoxifen should be stopped for 3 months before trying to conceive. Latest ESMO guidelines "strongly encourage the resumption of tamoxifen following delivery"1,27 It is also advised to continue active contraception up to 3 to 6 months following the last administered dose of anti-cancer therapy. ${ }^{27}$

Embryo or oocyte cryopreservation is the main method to preserve female fertility. ${ }^{36}$ Ovarian stimulation is carried out before commencing chemotherapy, but may result in relative delay in oncological treatment and increase serum oestradiol levels. This may be of concern in hormone-driven tumours like breast cancer. Laparoscopic ovarian tissue sampling and freezing before treatment are considered experimental. When needed, reimplantation of ovarian tissue in the pelvis after thawing may be a unique option for young girls with cancer. Over 60 pregnancies have been reported. ${ }^{37}$

\section{Conclusion}

The prognosis of PABC is similar to that of breast cancer in the non-pregnant state. Treatment should commence after diagnosis is established. Surgical treatment options are expanding and extensive data show that chemotherapy during pregnancy is safe and more options for treatment are now available. Whenever possible, the aim should be to carry the fetus to term. Future pregnancy is generally not contra-indicated.

\section{References}

1. RCOG Green-top guideline No. 12. Pregnancy and breast cancer. UK Royal College of Obstetricians and Gynaecologists; 2011.

2. Padmagirison R, Gajjar K, Spencer C. Management of breast cancer during pregnancy. Obstet Gynecol 2010;12:186-92.

3. Genin AS, Lesieur B, Gligorov J, Antoine M, Selleret L, Rouzier R. Pregnancy-associated breast cancers: do they differ from other breast cancers in young women? Breast 2012;21:550-5.

4. Lobiol S, Han SN, von Minchkitz G, et al. Treatment of breast cancer during pregnancy: an observational study. Lancet Oncol 2012;13:887-96.

5. Bell RJ, Fradkin P, Parathithasan N, Robinson PJ, Schwarz M, Davis SR. Pregnancy-associated breast cancer and 
pregnancy following treatment for breast cancer, in a cohort of women from Victoria, Australia, with a first diagnosis of invasive breast cancer. Breast 2013;22:980-5.

6. Borges VF, Schedin PJ. Pregnancy-associated breast cancer: an entity needing refinement of the definition. Cancer 2012;118:3226-8.

7. Walton JR, Prasad MR. Obstetric and neonatal outcomes of cancer treated during pregnancy. Clin Obstet Gynecol 2011;54:567-73.

8. Johansson AL, Andersson TM, Hsieh CC, et al. Stage at diagnosis and mortality in women with pregnancyassociated breast cancer (PABC). Breast Cancer Res Treat 2013;139:183-92.

9. Córdoba O, Llurba E, Saura C, et al. Multidisciplinary approach to breast cancer diagnosed during pregnancy: maternal and neonatal outcomes. Breast 2013;22:515-9.

10. Azim HA Jr, Peccatori FA. Treatment of metastatic breast cancer during pregnancy: we need to talk! Breast 2008;17:426-8.

11. McCready J, Arendt LM, Glover E, et al. Pregnancyassociated breast cancers are driven by differences in adipose stromal cells present during lactation. Breast Cancer Research 2014;16:R2.

12. Halaska MJ, Pentheroudakis G, Strnad P, et al. Presentation, management and outcome of 32 patients with pregnancyassociated breast cancer: a matched controlled study. Breast J 2009;15:461-7.

13. Rovera F, Frattini F, Coglitore A, et al. Breast cancer in pregnancy. Breast J 2010;16 Suppl 1:S22-5.

14. Viswanathan S, Ramaswamy B. Pregnancy-associated breast cancer. Clin Obstet Gynecol 2011;54:546-55.

15. Sánchez Martínez MC, Ruiz Simón A. Breast cancer during pregnancy. Breast Cancer Rest Treat 2010;123 Suppl 1:55-8.

16. International Commission on Radiological Protection. The 2007 recommendations of the International Commission on Radiological Protection. Ann ICRP 2007;37:1-332.

17. Beadle BM, Woodward WA, Middleton LP, et al. The impact of pregnancy on breast cancer outcomes in women $\leq 35$ years. Cancer 2009;115:1174-84.

18. Krishna I, Lindsay M. Breast cancer in pregnancy. Obstet Gynecol Clin North Am 2013;40:559-71.

19. Khera SY, KilukJV, Hasson DM, et al. Pregnancy-associated breast cancer patients can safely undergo lymphatic mapping. Breast J 2008;14:250-4.

20. Gentilini O, Cremonesi M, Trifirò G, et al. Safety of sentinel node biopsy in pregnant patients with breast cancer. Ann Oncol 2004;15:1348-51.

21. Gentilini O, Cremonesi M, Toesca A, et al. Sentinel lymph node biopsy in pregnant patients with breast cancer. Eur J Nucl Med Mol Imaging 2010;37:78-93.
22. International Commission on Radiological Protection. Pregnancy and medical radiation. Ann ICRP 2000;30:iiiviii,1-43.

23. Gropper AB, Calvillo KZ, Dominici L, et al. Sentinel lymph node biopsy in pregnant women with breast cancer. Ann Surg Oncol 2014;21:2506-11.

24. Karam A. Update on breast cancer surgery approaches. Curr Opin Obstet Gynecol 2013;25:74-80.

25. te Velde EA, Sonke G, Rutgers EJ. Breast cancer and pregnancy: diagnosis and treatment options. Breast Cancer Online 2009;12:e10.

26. Pruthi S, Haakkenson C, Brost BC, et al. Pharmacokinetics of methylene blue dye for lymphatic mapping in breast cancer-implications for use in pregnancy. Am J Surg 2011;201:70-5.

27. Peccatori FA, Azim HA Jr, Orecchia R, et al. Cancer, pregnancy and fertility: ESMO clinical practice guidelines for diagnosis, treatment and follow-up. Ann Oncol 2013;24 Suppl 6:vi160-70.

28. Lyman GH, Temin S, Edge SB, et al. Sentinel lymph node biopsy for patients with early-stage breast cancer: American Society of Clinical Oncology clinical practice guideline update. J Clin Oncol 2014;32:1365-83.

29. Azim HA Jr, Del Mastro L, Scargone G, Peccatori FA. Treatment of breast cancer during pregnancy: regimen selection, pregnancy monitoring and more... Breast 2011;20:1-6.

30. Cardonick E. Treatment of maternal cancer and fetal development. Lancet Oncol 2012;13:218-20.

31. Cardonick E, Bhat A, Gilmandyar D, Somer R. Maternal and fetal outcomes of taxane chemotherapy in breast and ovarian cancer during pregnancy: case series and review of the literature. Ann Oncol 2012;23:3016-23.

32. Mir O, Berveiller P. Increased evidence for use of chemotherapy in pregnancy. Lancet Oncol 2012;13:852-4.

33. Zagouri F, Sergentanis TN, Chrysikos D, et al. Taxanes for breast cancer during pregnancy: a systematic review. Clin Breast Cancer 2013;13:16-23.

34. Mir O, Berveiller P, Goffinet F, et al. Taxanes for breast cancer during pregnancy: a systematic review. Ann Oncol 2010;21:425-6.

35. Cardonick E, Gilmandyar D, Somer RA. Maternal and neonatal outcomes of dose-dense chemotherapy for breast cancer in pregnancy. Obstet Gynecol 2012;120:1267-72.

36. Angarita AM, Johnson CA, Fader AN, Christianson MS. Fertility preservation: a key survivorship issue for young women with cancer. Front Oncol 2016;6:102.

37. Donnez J, Dolmans MM. Ovarian cortex transplantation: 60 reported live births brings the success and worldwide expansion of the technique towards routine clinical practice. J Assist Reprod Genet 2015;32:1167-70. 